\title{
The evolution of V4334 Sgr (Sakurai's object) in the Mid-Infrared
}

\author{
H. U. Käufl ${ }^{1}$, J. Koller ${ }^{2,3}$, and F. Kerber ${ }^{4}$ \\ ${ }^{1}$ European Southern Observatory, Karl-Schwarzschild-Str. 2, 85748 Garching, Germany \\ 2 Department of Physics and Astronomy, MS 108, Rice University, 6100 S. Main St, Houston, TX 77005, USA \\ ${ }^{3}$ Los Alamos National Laboratory, X-1, PO Box 1663, MS P225, Los Alamos, NM 87545, USA \\ e-mail: jkoller@lanl.gov \\ ${ }^{4}$ Space Telescope European Coordinating Facility, Karl-Schwarzschild-Str. 2, 85748 Garching, Germany \\ e-mail: fkerber@eso.org ${ }^{\star}$
}

Received 25 February 2003 / Accepted 15 May 2003

\begin{abstract}
Since Sakurai's object (V4334 Sgr) underwent a late helium flash in 1995, its fast evolution has been monitored with great effort. We present TIMMI and TIMMI 2 photometric observations covering a spectral range from 5 to $20 \mu \mathrm{m}$ with a total of 8 filters. We found drastic changes in the spectral energy distribution indicating a drop of temperature in the dust envelope from 1000 to $600 \mathrm{~K}$ in half a year only. Combined with optical observations reported elsewhere, we conclude that the phase of dust formation has probably stopped in mid-2001 and that the dusty envelope has been expanding only, ever since. An expansion velocity of about $1000 \mathrm{~km} \mathrm{~s}^{-1}$ is required to explain the rapid cooling. Other optical and near-infrared observations support the existence of such high velocity flows in the envelope of V4334 Sgr. An estimate for the bolometric luminosity of $\approx 2000 L_{\odot}$ is derived for a distance of $1 \mathrm{kpc}$. Taking into account theoretical models a distance of $2 \pm 1 \mathrm{kpc}$ to V4334 Sgr is suggested. As a consequence of its fast final helium flash evolution, Sakurai's object may start to destroy its surrounding dust in the not too distant future.
\end{abstract}

Key words. stars: AGB and post-AGB - stars: evolution - circumstellar matter - planetary nebulae: individual: Sakurai's Object (V4334 Sgr)

\section{Introduction}

Sakurai's object (V4334Sgr) was discovered as a slow Nova in February 1996. Follow-up observation established that this is the first case of a final helium-flash sinceV605 Aql in 1919 (Duerbeck \& Benetti 1996; Kerber 2001). FGSge probably also is a final helium shell flash object, but has shown a much slower evolution observed over the past 100 years; see e.g., Jorcsik \& Montesinos (1999) for a review of relevant observations and the evolution. Now of course, Sakurai's object is the best studied case and the only one that has been observed at wavelengths other than optical during or shortly after the flash. The observed evolution suggests the following sequence of events: V4334 Sgr was an evolved very hot (>100000 K) central star of a PN (Kerber et al. 1999a; Pollacco 1999). In 1995, a few thousand years after the nebula had formed, the central star underwent a very late helium flash. This sent the star back to the asymptotic giant branch (AGB), increasing drastically in size on its way through the HR-diagram. This cool "born-again" giant showed fast and massive changes in its spectrum as carbon bearing molecules formed more than a

\footnotetext{
Send offprint requests to: H. U. Käufl, e-mail: hukaufl@eso.org

$\star$ Based on observations collected at the European Southern Observatory, Chile, proposals 65.L-0702, 67.D-0413, 68.D-0542.
}

year after the actual helium flash (Asplund et al. 1997) very much comparable to V605 Aql (Clayton \& De Marco 1997). Shortly thereafter, a strong infrared excess (Kimeswenger et al. 1997) developed as a direct result of mass loss and associated dust formation (Kerber et al. 1997). Using general and discretionary time on the Infrared Space Observatory (ISO), we employed ISOCAM's full LW wavelength range (4 to $15 \mu \mathrm{m}$ ) (Cesarsky et al. 1996) to monitor the evolution of Sakurai's object for a full year from February 1997 to 1998. The flux increased by a factor of ten during that time. The most likely explanation was that very hot dust had recently formed in an extended, optically thin shell around the star (Kerber et al. 1999b). Subsequently V4334 Sgr showed significant fadings in the visual brightness as our line of sight became optically thick. A very similar behavior is well known from a special kind of irregular variables, the R CrB stars (Clayton 1996). Another interesting object which recently underwent an outburst in luminosity is V838 Mon. Although it probably is not a He-flash object, its nova-like eruption was followed by the development of an IR-excess due to the formation of a dust shell (Käufl et al. 2002; Crause et al. 2003). In contrast to Sakurai's object, V838 Mon during outburst showed P Cygni profiles indicating very significant velocities of circumstellar matter at that stage. The nature of V838 Mon is still under debate. 
Table 1. TIMMI observations of Sakurai's object. Flux values are in Jansky and wavelengths in $\mu \mathrm{m}$.

\begin{tabular}{lrrrrrr}
\hline \hline Date & \multicolumn{2}{c}{$F_{v}[\mathrm{Jy}]$} & & & & \\
& $\lambda(\mu \mathrm{m})=7.7$ & 8.4 & 9.5 & 9.8 & 11.5 & 12.8 \\
\hline \multirow{2}{*}{ 8 Mar. 98 } & 8.7 & 21.58 & 14.26 & 14.98 & 8.31 & 8.63 \\
\hline
\end{tabular}

As the mass loss in V4334 Sgr continued, it became very faint in the visual (>20 mag) in 1999 and has remained so because it is "buried" in its own dust.

Therefore, even $8 \mathrm{~m}$ class telescopes cannot reveal the evolution of the photosphere which is hidden behind the dust envelope. In the mid-infrared (MIR) of course, circumstellar extinction is negligible. The observed photons are the result of processing of the radiation, which originally has been emitted from the photosphere, by the dusty material around the central star. Monitoring this wavelength region is thus of crucial importance for following the evolution of circumstellar matter and its properties. Here we present the first results from our ground-based MIR monitoring over the last years covering the wavelength range from 5 to $20 \mu \mathrm{m}$. Recent evolutionary calculations (Herwig 2001; Lawlor \& MacDonald 2003) clearly suggest that the star will not stay in the born-again giant phase for very long. It is expected that within a few decades it will leave the AGB, heat up and retrace its own evolution towards the planetary nebula phase for a second time.

\section{Observations and data reduction}

The observations were done using the ESO facility instruments TIMMI (Käufl et al. 1994) and TIMMI 2 (Reimann et al. 2000). Both instruments are cryogenic infrared focal reducers following an identical concept, optimized for the wavelength interval $8-13 \mu \mathrm{m}$ (atmospheric $N$-band). However, vast technical differences in these instruments required a complete change of the filter complement. Unfortunately, the filter pass-bands for the two instruments are slightly different and only TIMMI 2 offers access to the wavelength regime $\lambda \geq 18 \mu \mathrm{m}$.

For background reduction the classical infrared chopping and nodding technique has been employed. The resulting images were evaluated by applying standard aperture integration with $4^{\prime \prime}$ diameter.

For flux calibration usually the star HD 169916 ( $\lambda$ Sgr, spectral type K1 IIIb) has been used. The spectral energy distribution was taken from Cohen (1998). The statistical error of the photometry is $2 \%$ and absolute errors $<5 \%$, in general. Nevertheless, glitches due to bad weather, have occurred and are discussed in the text.

The 2.5 year gap between March 1998 and October 2000 was imposed by technical constraints in the change-over from TIMMI to the next generation instrument TIMMI 2. The October 2000 measurements were in fact done during daytime immediately after first light of TIMMI 2 at La Silla (Käufl et al. 2000).
Table 2. TIMMI 2 observations of Sakurai's object. Flux values are in Jansky and wavelengths in $\mu \mathrm{m}$.

Note: Wavelengths provided here are the effective wavelengths of the filters, not to be confused with the ESO fits-keyword filter names used elsewhere in the text.

\begin{tabular}{|c|c|c|c|c|c|c|c|c|}
\hline \multirow[t]{2}{*}{ Date } & \multicolumn{2}{|l|}{$F_{v}[\mathrm{Jy}]$} & \multirow[b]{2}{*}{8.6} & \multirow[b]{2}{*}{9.5} & \multirow[b]{2}{*}{10.2} & \multirow[b]{2}{*}{11.6} & \multirow[b]{2}{*}{12.2} & \multirow[b]{2}{*}{20.0} \\
\hline & $\lambda(\mu \mathrm{m})=4.7$ & 7.7 & & & & & & \\
\hline 11 Oct. 00 & $\ldots$ & 69.1 & 66.0 & 45.3 & 44.6 & 35.6 & 32.9 & \\
\hline 11 May 01 & 29.7 & 47.8 & 55.1 & 48.0 & 49.8 & 51.9 & 49.4 & . \\
\hline 16 Jun. 01 & 31.7 & 52.8 & 50.9 & 51.4 & 56.8 & 56.4 & 53.4 & \\
\hline 13 Aug. 01 & $\ldots$ & 67.0 & 64.6 & 60.9 & 68.9 & 57.3 & 56.5 & 27.2 \\
\hline \multirow[t]{2}{*}{08 Oct. 01} & $\ldots$ & $\ldots$ & (27.8) & 63.1 & 55.6 & $\ldots$ & 52.5 & $\ldots$ \\
\hline & $\ldots$ & 62.2 & 56.7 & 55.0 & 56.7 & 61.1 & 66.5 & . \\
\hline
\end{tabular}

As the complete filter set of TIMMI 2 was not available from the beginning, it was not possible to have the same filter complement throughout the entire monitoring program.

\section{Results and dust evolution}

\subsection{Mar. 8th, 1998}

In continuation of the ISO observations we measured the flux of Sakurai's object with TIMMI at the $3.6 \mathrm{~m}$ telescope at ESO in La Silla in August 1998 (Table 1). We used the 7.7, 8.4, 9.5, 9.8, and $11.5 \mu \mathrm{m}$ filters in order to obtain the spectral energy distribution (SED). For an optically thick shell with a sharp, fixed outer edge the slope would be of the form $\lambda F \propto \lambda^{-\alpha}$. For very large $\lambda$, one gets $\alpha \approx 3$. However, for the observed wavelengths one expects a slope $\alpha<3$. In Mar. 1998 we find a slope of $\alpha \approx 3.3$, decidedly larger than expected and an indication of significant broadband absorption.

\subsection{Oct. 11 th, 2000}

The data set was obtained with TIMMI 2 at 7.9, 8.6, 9.8, 10.4, 11.9 , and $12.9 \mu \mathrm{m}$ (Table 2). In just two years, Sakurai's object had changed dramatically. The peak of the SED shifted to longer wavelengths due to expansion of the dust shell. A blackbody fit to these October data yield approximately $950 \mathrm{~K}$ which means that since 1998 it had only marginally cooled. Lynch et al. (2002) report a temperature of $1000 \mathrm{~K}$ for Sept. 1998. The spectral slope changed to $\alpha \approx 2.8$.

We also observe an absorption feature at $9.8 \mu \mathrm{m}$ (depth $8 \%$ ) (Fig. 1). Lynch et al. (2002) report data from Sept./Oct. 1998. In their Fig. 1a small absorption feature is indicated but not discussed as such. In a very recent paper, Evans et al. (2002) interpret such a feature in their spectrum taken in July 2002 as being an interstellar $9.7 \mu \mathrm{m}$ absorption feature of amorphous (i.e. non-crystalline) silicates (Lutz et al. 1996). A more detailed discussion about the behaviour of the absorption feature will be given in a subsequent paper covering our recent spectra. 


\subsection{May 11th 2001 and June 16th, 2001}

The same filters with TIMMI 2 were used as in Oct. 2000 but including the $4.7 \mu \mathrm{m}$ filter. Again, the spectral energy distribution changed drastically. The flux increased strongly at 10.4 , 11.9 , and $12.9 \mu \mathrm{m}$ but decreased at shorter wavelength due to strong extinction in the mid and near-infrared (MN-IR). Lederle \& Kimeswenger (2001) confirm this with $I, J, K$ magnitudes measured a few months earlier (Feb. 18, 2001). A blackbody fit suggests a dramatic change, too: now the temperature is about $600 \mathrm{~K}$. This drastic change in temperature from $1000 \mathrm{~K}$ to $600 \mathrm{~K}$ in only half a year is probably due to strong cooling without new dust formation. The dust envelope around Sakurai's object consists of multiple shells with different temperature components which are simultaneously expanding and cooling. Such multiple dust shells have been modeled by Kimeswenger \& Koller (2002).

Kerber et al. (2002) recently discovered emission from $[\mathrm{NII}],[\mathrm{OI}],[\mathrm{OII}]$ and faint $\mathrm{H} \alpha$ around the core of V4334 Sgr indicating the presence of freshly ionized matter. The cause of ionization could not be determined conclusively, both, shocks from the observed high velocity $(-350 / 200 \pm$ $50 \mathrm{~km} \mathrm{~s}^{-1}$ ), or an increase of the photospheric temperature to at least $20000 \mathrm{~K}$ could account for the observed ionized matter. Regardless of the relative importance of the two processes, it is likely that conditions for the formation of dust close to the star's photosphere no longer persist, and the formation of dust had come to an end by mid-2001. Therefore, the outer shells are expanding and cooling while the supply of hot, freshly formed dust has ceased. We created a simple model based on the equilibrium dust temperature of $950 \mathrm{~K}$ in Oct. 2000 and $600 \mathrm{~K}$ in June 2001. Our calculations assume a thin dust shell containing an averaged particle size of $23 \AA$. The equilibrium temperature is almost independent of the luminosity but falls off $\propto r^{-1 / 2}$ (Koller 2000, 2002). We find that in order for the dust to cool from $950 \mathrm{~K}$ to $600 \mathrm{~K}$ in only 8 months, an expansion velocity of at least $1000 \mathrm{~km} \mathrm{~s}^{-1}$ is required. The existence of such large velocities is, corroborated by the observation of a P Cygni profile of the He I line at $1.083 \mu \mathrm{m}$ (Eyres et al. 1999; Geballe et al. 2002) which indicates an expansion velocity as fast as $800 \mathrm{~km} \mathrm{~s}^{-1}$. Also the $\approx \pm 300 \mathrm{~km} \mathrm{~s}^{-1}$ observed by Kerber et al. (2002) are a lower limit due to the geometry of the bipolar outflows. Using a simple model they find the true outflow velocity to be probably around $800 \mathrm{~km} \mathrm{~s}^{-1}$.

\subsection{Aug. 13th, 2001}

The TIMMI 2 measurements on Aug. 13th, 2001 were made with the same filters as in Oct. 2000 plus the $20 \mu \mathrm{m}$ filter. The flux in filters 7.9 and $8.6 \mu \mathrm{m}$ returned to the values from Oct. 2000, whereas the flux increased by almost $60 \%$ at longer wavelengths, confirming the continued expansion of the ejecta.

\subsection{Oct. 8th, 2001}

This data set was obtained on Oct. 8th, 2001 using the 8.9, 9.8, 10.4 and $12.9 \mu \mathrm{m}$ filters in TIMMI 2 (Table 2). Additionally, a specialized filter for the $12.8 \mu \mathrm{m}$ transition of [Ne II] was

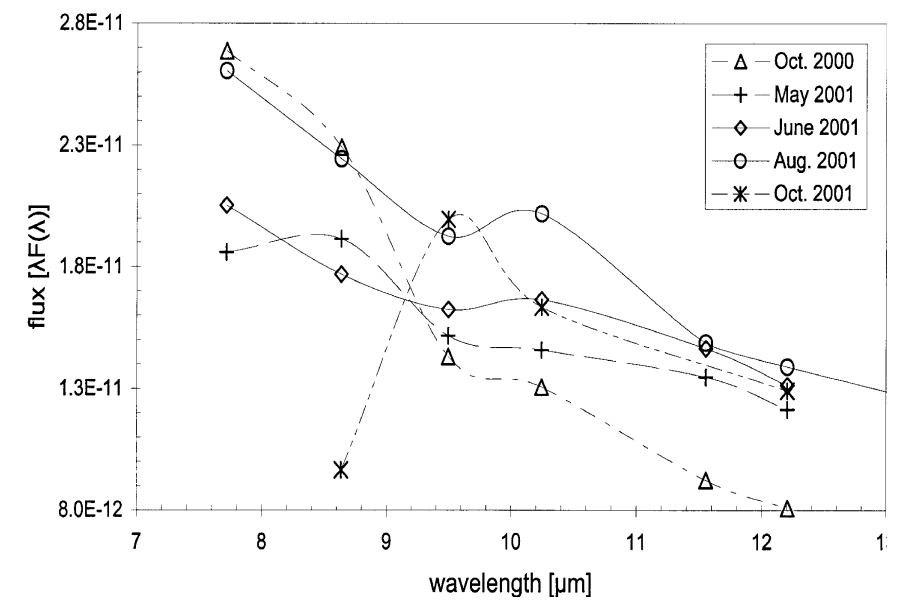

Fig. 1. SED of Sakurai's object between Oct. 2000 and 2001 at MIR wavelengths of 7 to $13 \mu \mathrm{m}$.

available for the first time. The $Q$-band measurements could not be repeated as the filter we used in August 2001 is extremely dependent on atmospheric water vapor content. In fact observing conditions were not exactly photometric. Therefore, the dip in the $8.9 \mu \mathrm{m}$ filter should not be over interpreted. It is most likely a glitch in the data. The deep search in the [Ne II] filter did not show any indication of line emission from ionized matter.

\section{Dust shell model}

We used a recently developed code NILFISC (Koller \& Kimeswenger 2001a) to model the circumstellar dust of Sakurai's object. The code applies a grain-size distribution and stochastic heating of small dust particles (Guhatakurta \& Draine 1989) to allow for temperature fluctuations. However, currently temperature fluctuations don't play a major role in Sakurai's object because the density of the radiation field is too high. Only later, when the dust shell will have expanded to a larger size $\left(r \approx 10^{14} \mathrm{~m}\right)$, temperature fluctuation of small dust particles will become important, noticeable by an increased near infrared (NIR) flux. Our model assumed a dust shell with a decreasing density profile $\propto 1 / r^{2}$ consisting of carbonaceous grains. The absorption and scattering coefficients for these grains were taken from Laor \& Draine (1993). We assumed a simple blackbody radiation field to heat the dust particles. As discussed by Koller \& Kimeswenger (2001a, 2001b) a simple blackbody radiation field for the central star is sufficient. The radiation field from a $\mathrm{H}$-poor star with the same total luminosity does not change the dust emission significantly. We applied a power law distribution (Mathis et al. 1977) of dust grains from 6-1700 $\AA$ to fit the spectrum from May 2001 (Fig. 2). However, we used only carbonaceous grains for our calculations, and a different chemistry might play an important role for the evolution of the dust.

We also observed that from Oct. 11, 2000 to June 16, 2001 the color between wavelength $11.4 \mu \mathrm{m}$ and $12.9 \mu \mathrm{m}$ (Fig. 3 ) did not change. This suggests that these wavelengths were not contaminated by effects like dust formation or significant cooling but only expansion. The luminosity at those wavelengths 


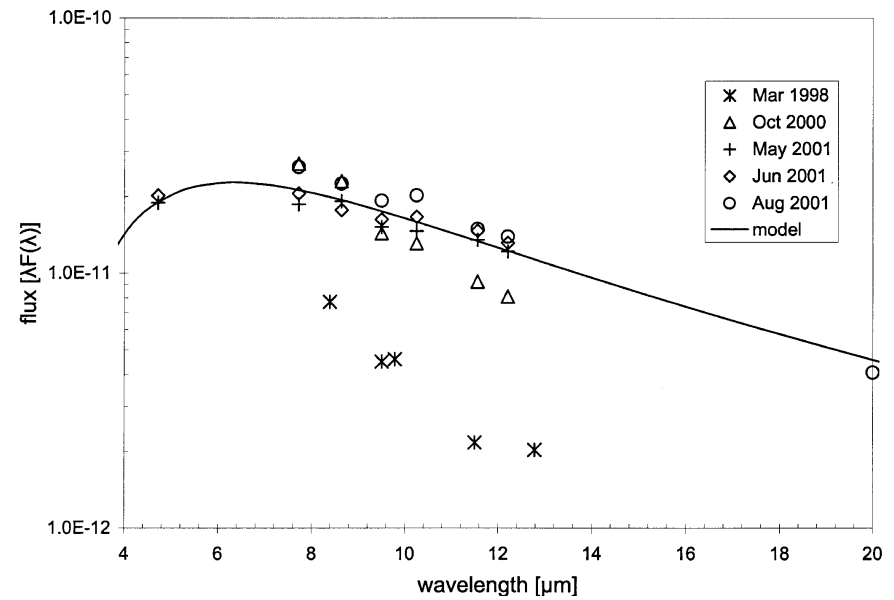

Fig. 2. Sakurai's object data from 1998 to 2001 including model calculations for May 2001 data using a grain size distribution from $6-1700 \AA$. The model was constrained in the near infrared by $I$, $J, K$ measurements by Lederle \& Kimeswenger (2001).

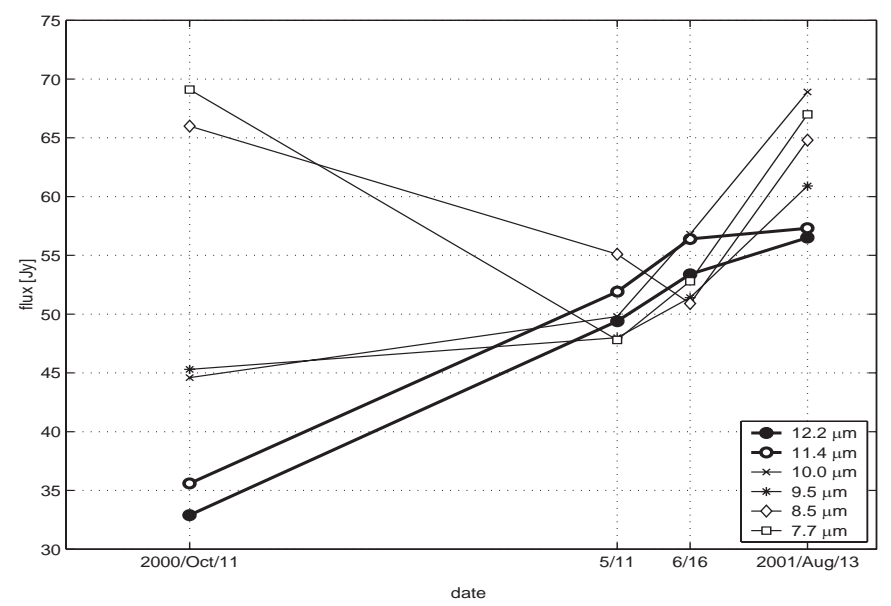

Fig. 3. Time evolution Sakurai's object's flux in several filters. Central wavelengths of the filters are given in $\mu \mathrm{m}$ and fluxes are in Jansky. Note the almost parallel evolution at $11.4 \mu \mathrm{m}$ and $12.9 \mu \mathrm{m}$.

increased almost linearly by a factor of 1.6. Using $F_{1}(\lambda) / F_{2}(\lambda) \propto r_{1}^{2} / r_{2}^{2}$, we find that the dust shell expanded by $r_{1}^{2} / r_{2}^{2}=\sqrt{1.6}$.

To obtain an absolute radius at a given time, we can use the expansion velocity of Sakurai's object. Geballe et al. (2002) report a significant P Cygni profile for helium at $1.083 \mu \mathrm{m}$ with velocities between $500-800 \mathrm{~km} \mathrm{~s}^{-1}$. These velocities were observed for 1999. Since high velocities have also been found by Kerber et al. (2002) for mid-2001, we assume the velocity has remained constant during that period; we then obtain $\Delta r=1-1.7 \times 10^{13} \mathrm{~m}$. Now, the system of two equations can be solved for an absolute radius at a given time. We find that in the beginning of October 2000, the shell size was $4-6 \times 10^{13} \mathrm{~m}$ depending on the exact expansion velocity $500-800 \mathrm{~km} \mathrm{~s}^{-1}$.

\section{Bolometric luminosity and distance}

Were the dust shell around V4334 Sgr perfectly spherical, then it could be considered a calorimeter. The dust is intercepting all optical radiation extremely effectively and the energy is re-radiated from the warm dust. It is obvious, that along the line of sight towards V4334 Sgr the dust is optically thick for the primary radiation of the central star. However, recent optical data by Kerber et al. (2002) indicate a more complex situation: the dust envelope around the star does not cover $4 \pi \mathrm{sr}$ but is more likely of toroidal shape. For the following it is assumed, that the toroidal dust envelope intercepts $50 \%$ of the optical radiation. From a simple integration of the Planck function $\left(\frac{\mathrm{d}^{3} P}{\mathrm{~d} A \mathrm{~d} \Omega \mathrm{d} \lambda}\right)$ for the TIMMI2 filter passbands one can derive an interesting feature for any $10 \mu \mathrm{m}$ camera observing a black body source in the temperature range $250-800 \mathrm{~K}$ : the fraction of the total energy detected in the TIMMI $210 \mu \mathrm{m}$ filters hardly changes with temperature.

$\int_{\lambda_{1}}^{\lambda_{2}} \frac{\mathrm{d}^{3} P}{\mathrm{~d} A \mathrm{~d} \Omega \mathrm{d} \lambda} \mathrm{d} \lambda / \int_{0}^{\infty} \frac{\mathrm{d}^{3} P}{\mathrm{~d} A \mathrm{~d} \Omega \mathrm{d} \lambda} \mathrm{d} \lambda \approx 0.035-0.07$.

With the assumptions on geometry made above it is then obvious that e.g. the flux detected in the $8.6 \mu \mathrm{m}$ filter $\left(\lambda_{1}=8.29 \mu \mathrm{m}\right.$, $\left.\lambda_{2}=9.07 \mu \mathrm{m}\right)$ is $1.7-3.5 \%$ of the entire bolometric flux. From the photometry in Table 2, with a geometrical correction factor of 2 (see above) we thus derive an estimate for $L_{\text {bolo }}$ of $2000 L_{\odot}$ for a distance of $1 \mathrm{kpc}$. The error of this number is $\approx 20 \%$ taking into account uncertainties in the dust spectrum. The uncertainty of the geometrical correction may account for a factor of 2 .

Recent theoretical evolutionary modelling by Lawlor \& MacDonald (2003), who present stellar evolution models for a star with a main-sequence mass of $1 M_{\odot}$, gives a theoretical luminosity for the AGB phase of order of $4-6 \times 10^{3} L_{\odot}$. Assuming that the mass estimate is a lower limit we assume a somewhat higher luminosity. We conclude that the bolometric luminosity is compatible with a distance of $2 \pm 1 \mathrm{kpc}$.

\section{Conclusions}

After ISO, we continued to monitor the changes of Sakurai's object at MIR wavelengths with TIMMI and TIMMI 2 at ESO. The changes in the SED are profound. The star clearly produced copious amounts of dust while expanding rapidly. During this rapid expansion the newly formed dust cools, thus shifting the spectrum to longer wavelengths, while the formation of hot dust continued. We modeled the expanding dust shell and found that a size distribution of carbonaceous grains from $6 \AA$ to $0.2 \mu \mathrm{m}$ fits the spectrum reasonably well. Furthermore, multiple dust formation events are required to model Sakurai's object's frequent changes as Kimeswenger \& Koller (2002) showed with earlier data. Also a distribution of grain sizes is necessary to explain the deviations from a simple blackbody slope of an optically thick shell.

Recent VLT observations by Kerber et al. (2002) have revealed the existence of freshly ionized matter in high velocity outflows. This indicates that conditions may no longer have been favourable for dust formation by mid-2001, which is in good agreement with our finding in the MIR of a rapidly decrease of the average dust temperature. At this point the star may already be heating up. In the optical, which is still affected by significant circumstellar extinction no lines requiring excitation energies of more than $15 \mathrm{eV}$ have been observed. 
Therefore, the [Ne II] line at $12.8 \mu \mathrm{m}$ (excitation energy approx. $21.6 \mathrm{eV}$ ), where extinction is minimal, will be an excellent indicator of future temperature changes. Theoretical models (Lawlor \& MacDonald 2003) suggest that V4334 Sgr will start to heat up again only a few decades after reaching the AGB. They also show that the lightcurves of V4334 Sgr, V605 Aql and FG Sge can be explained by a single model using reduced convective mixing efficiency, which leads to a double loop in the H-R diagram. Therefore all three may be closely related objects at different stages of their final helium shell flash evolution. An increase in the temperature of the central star, of course, results in an increase in the number of high energy photons which in turn will lead to the destruction of dust particles, preferentially small ones at the inner shell boundary. This will have a fundamental effect on the grain size distribution and the SED in the MIR.

Sakurai's object will thereby turn from a dust producer to a dust destroyer and may emerge from its dusty cocoon in the not too distant future. This makes continued monitoring - in the MIR and other wavelength regions - all the more important. Particularly interesting here is that the velocity dispersion measured by Kerber et al. (2002), $1500 \mathrm{~km} \mathrm{~s}^{-1}$ for the [N II] line at $658.3 \mathrm{~nm}$, in combination with our distance estimate suggests an expansion of the object of $\approx 0.2^{\prime \prime}$ per year. This makes V4334 Sgr an extremely interesting target for VLT/VLTI observations in the MIR.

Acknowledgements. We thank all members of ESO's $3.6 \mathrm{~m}$ Team for their excellent work during the service mode observations.

\section{References}

Asplund, M., Gustafsson, B., Lambert, D. L., \& Kameswara Rao, N. 1997, A\&A, 321, L 17

Cesarsky, C. J., Abergel, A., Agnes̀e, P., et al. 1996, A\&A, 315, L 32

Clayton, G. C. 1996, PASP, 108, 225

Clayton, G. C., \& De Marco, O. 1997, AJ, 114, 2679

Cohen, M. 1998, AJ, 115, 2092
Crause, L. A., Warrick, A. L., Kilkenny, D., et al. 2003, MNRAS, in press

Duerbeck, H. W., \& Benetti, S. 1996, ApJ, 468, L111

Evans, A., Geballe, T. R., Smalley, B., Tyne, V. H., \& Eyres, S. P. S. 2002, A\&A, 394, 971

Eyres, S. P. S., Smalley, B., Geballe, et al. 1999, MNRAS, 307, L11

Geballe, T. R., Evans, A., Smalley, B., Tyne, V. H., \& Eyres, S. P. S. 2002, Ap\&SS, 279, 39

Guhathakurta, P., \& Draine, B. T. 1989, ApJ, 345, 230

Herwig, F. 2001, ApJ, 554, L71

Jorcsik, J., \& Montesinos, B. 1999, New Astron. Rev., 43, 415

Käufl, H. U., Jouan, R., Lagage, P. O., et al. 1994, Infared Phys. Technol., 35, 203

Käufl, H. U., Ageorges, N., Dietzsch, E., et al. 2000, ESO Messenger, 102,4

Käufl, H. U., Kerber, F., \& Koller, J. 2001, IAU Circ., 7725

Käufl, H. U., Locurto, G., Kerber, F., \& Heijligers, B. 2002, IAU Circ., 7831

Kerber, F., Gratl, H., Kimeswenger, S., \& Roth, M. 1997, Ap\&SS, 255, 279

Kerber, F., Blommaert, J. A. D. L. S., Kimeswenger, S., et al. 1999b, A\&A, 350, L 27

Kerber, F., Köppen, J., Roth, M., \& Trager, S. C. 1999a, A\&A, 344, L 79

Kerber, F. 2001, Ap\&SS, 275, 91

Kerber, F., Pirzkal, N., De Marco, O., et al. 2002, ApJ, 581, L 39

Kimeswenger, S., Gratl, H., Kerber, F., et al. 1997, IAU Circ., 6608

Kimeswenger, S., \& Koller, J. 2002, Ap\&SS, 279, 149

Koller, J. 2000, MSc. Thesis, University of Innsbruck

Koller, J., \& Kimeswenger, S. 2001a, ApJ, 559, 419

Koller, J., \& Kimeswenger, S. 2001b, Ap\&SS, 275, 212

Koller, J. 2002, Rev. Mex. Conf., 12, 165

Laor, A., \& Draine, B. T. 1993, ApJ, 402, 441

Lawlor, T. M., \& MacDonald, J. 2003, ApJ, 583, 913

Lederle, C., \& Kimeswenger, S. 2001, IAU Circ., 7592

Lutz, D., Feuchtgruber, H., Genzel, R., et al. 1996, A\&A, 315, L 269

Lynch, D. K., Rudy, R. J., Russell, R., et al. 2002, Ap\&SS, 279, 57

Mathis, J. S., Rumpl, W., \& Nordsieck, K. H. 1977, ApJ, 217, 425

Pollacco, D. 1999, MNRAS, 304, 127

Reimann, H.-G., Linz, H., Wagner, R., et al. 2000, Proc. SPIE, 4008, 1132, Optical and IR Telescope Instrumentation and Detectors, ed. M. Iye, \& A. F. Moorwood 